Revue internationale de l'économie sociale

Recma

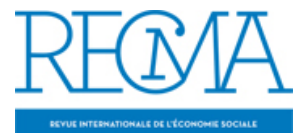

\title{
Formations en économie sociale et solidaire : l'action de la DIES
}

\section{Scarlett Courvoisier}

Numéro 281, juillet 2001

Économie sociale et/ou solidaire?

URI : https://id.erudit.org/iderudit/1024024ar

DOI : https://doi.org/10.7202/1024024ar

Aller au sommaire du numéro

Éditeur(s)

Institut de l'économie sociale (IES)

ISSN

1626-1682 (imprimé)

2261-2599 (numérique)

Découvrir la revue

Citer cet article

Courvoisier, S. (2001). Formations en économie sociale et solidaire : l'action de la DIES. Revue internationale de l'économie sociale, (281), 98-100.

https://doi.org/10.7202/1024024ar d'utilisation que vous pouvez consulter en ligne. 


\section{FORMATIONS EN ÉCONOMIE SOCIALE ET SOLIDAIRE : \\ L'ACTION DE LA DIES}

(*) Scarlett Courvoisier est conseillère technique à la Délégation interministérielle. par Scarlett Courvoisier ${ }^{*}$

$\mathrm{L}$

a question de l'information et de la formation à l'économie sociale et solidaire est à inscrire dans le cadre de la politique territoriale du secrétariat d'Etat à l'économie solidaire et de la DIES qui a été lancée par les consultations régionales de l'économie sociale et solidaire, puis par l'appel à projets « Dynamiques solidaires » en 2000.

Rappelons brièvement les principaux axes et faits dessinant le cadre de cette politique : l'application et le suivi de la circulaire du 22 décembre 1999 relative aux relations de l'Etat avec les associations dans les départements ; la circulaire du 5 septembre 2000 Voynet-Hascoët relative à la place de l'économie sociale et solidaire dans les processus de contractualisation territoriale ; la circulaire du $1^{\text {er }}$ décembre 2000 sur les conventions pluriannuelles d'objectifs ; le projet en cours d'une circulaire relative au renforcement du rôle et des missions des correspondants régionaux ; la signature en cours d'une dizaine de conventions pluriannuelles d'objectifs avec les chambres régionales de l'économie sociale ; enfin, au-delà des contrats de plan Etat-régions, des moyens budgétaires nouveaux pour les correspondants et les délégué(e)s départementaux à la vie associative (moyens de services et appels à projets " Dynamiques solidaires »).

Organisés à l'initiative de la DIES dans le cadre de cette politique d'animation territoriale, deux programmes d'information-formation, lancés en 2001, se déroulent actuellement en régions.

\section{" Rencontres des acteurs publics et des acteurs de la société civile sur l'économie sociale et solidaire "}

Il s'agit d'une information-formation destinée aux services déconcentrés de l'Etat avec les responsables, " têtes de réseau " de l'économie sociale et solidaire, intervenant dans chaque région au cours de l'année 2001.

Les objectifs sont les suivants :

- faciliter une meilleure connaissance et reconnaissance mutuelle ;

- rapprocher les agents des différents départements ministériels chargés d'appliquer les politiques contractuelles, et ces derniers des acteurs de la société civile situés dans le champ de l'économie sociale et solidaire ; 
- répondre à des besoins et à des demandes d'informations enregistrés sur le terrain de part et d'autre ;

- aider au projet territorial de l'Etat et à la réforme de l'Etat, notamment en termes de meilleures coordination, concertation et interministérialité, pour un meilleur service public rendu aux usagers et citoyens que sont les partenaires de l'Etat dans les secteurs, par définition transversaux, de l'économie sociale et solidaire.

En concevant et en organisant au niveau régional, en 2000, des consultations régionales de l'économie sociale et solidaire, la DIES a aidé aux rapprochements de ces divers acteurs en invitant les services déconcentrés de l'Etat. La même année, le secrétariat d'Etat à l'Economie solidaire, à travers l'appel à projets " Dynamiques solidaires ", a facilité le rapprochement des services déconcentrés de l'Etat pour avis et instruction des dossiers dans le cadre de comités régionaux.

Le programme de formation, dans la même logique, entend consolider les acquis et renforcer cette démarche basée sur une meilleure compréhension mutuelle des logiques d'action, de compétences et de territoires des uns et des autres. Il s'agit de créer un processus d'acquisition progressive d'une culture commune sur l'économie sociale et solidaire et des conditions favorables à un meilleur dialogue entre les services déconcentrés de l'Etat et leurs partenaires. En effet, au cours de ces rencontres, un dialogue est organisé sur la base de thématiques convergentes et partagées.

\section{La mise en œuvre}

- En amont, une convention a été passée avec l'Institut d'études politiques de Grenoble par la DIES pour concevoir l'économie générale du projet et lancer une phase test, animée par une universitaire de Grenoble, Danièle Demoustier. Elle s'est déroulée en Paca (région), Picardie (région et départements) et dans le département de l'Isère, afin notamment d'apprécier le niveau administratif et géographique le plus pertinent.

- Une réunion d'évaluation a eu lieu en mars, avec un collectif d'universitaires, avant lancement du programme à l'ensemble des régions.

- Le travail en région nécessite trois temps après accord du préfet (les réunions étant placées sous son autorité et organisées par les correspondants régionaux de la DIES sous la responsabilité du Secrétariat général pour les affaires régionales (SGAR) :

- une réunion d'information sur le projet lui-même, animée par la DIES - une réunion préparatoire à laquelle assiste la DIES et un universitaire pour travailler sur des fiches, déterminer les thématiques de préoccupations convergentes et les futures intervenants ;

- la rencontre informative elle-même, animée par l'universitaire, doit déterminer en conclusion des chantiers de préoccupations communes à l'ensemble des acteurs pour améliorer l'existant.

Aujourd'hui, sept régions, outre les régions-tests, ont réalisé la première réunion : Bretagne, Nord-Pas-de-Calais, Corse, Auvergne, LanguedocRoussillon, Basse-Normandie, Rhône-Alpes. 


\section{Un programme de formation à destination des délégué(e)s départementaux à la vie associative}

La circulaire du 22 décembre 1999 relative aux relations de l'Etat avec les associations dans les départements a confié à la DIES un rôle d'animation et de coordination de l'ensemble des délégué(e)s départementaux à la vie associative. Outre deux réunions nationales par an pour l'ensemble de ces agents dont la vocation interministérielle a été affirmée dans cette circulaire, la DIES organise en liaison avec le ministère de la Jeunesse et des Sports un programme de formation interrégional et interdépartemental sur deux journées en sept sessions. La première journée est centrée sur les enjeux et les problématiques actuels du monde associatif; elle fait intervenir des représentants des CPCA régionales, du CNVA, de la Fonda... La deuxième journée rassemble des interventions plus techniques : fiscales, juridiques... Ces deux journées ont pour objectifs de donner aux DDVA une information très actualisée, des compétences élargies à l'ensemble des divers secteurs du monde associatif, et d'aider à la mise en synergie des compétences. Aujourd'hui, trois sessions ont été réalisées et le programme devra se terminer à la fin de 2001. 